\title{
Forma tumoral da esquistossomose mansoni cerebelar: relato de caso e medida dos granulomas
}

\author{
The tumoral form of cerebellar schistosomiasis: \\ case report and measure of granulomas
}

\author{
Pedro Raso ${ }^{1}$, Alexandre Tafuri ${ }^{2}$, Ney da Fonseca Lopes ${ }^{3}$, \\ Eduardo Rossi Monteiro ${ }^{4}$ e Wagner Luiz Tafuri ${ }^{5}$
}

\begin{abstract}
RESUMO
Caso raro de forma tumoral da esquistossomose mansoni cerebelar diagnosticada pela biópsia, em um paciente de 15 anos, que apresentou sinais e sintomas neurológicos 60 dias antes da cirurgia. A tomografia computadorizada revelou lesão expansiva, hiperdensa, localizada no cerebelo, sugestiva de glioma. O exame histopatológico mostrou numerosos ovos de $\mathrm{S}$. mansoni envolvidos por reação inflamatória granulomatosa na fase necrótico-exsudativa, confluentes, localizados principalmente na camada interna, granular, do cerebelo, formando pseudotumor no verme cerebelar e hemorragia recente na ponte.Foram medidas as áreas dos granulomas.
\end{abstract}

Palavras-chaves: Schistosoma mansoni. Esquistossomose cerebelar. Tumor cerebelar. Neuro-esquistossomose mansoni.

\begin{abstract}
An unusual case of the tumoral form of cerebellar Schistosomiasis mansoni, in a 15 year-old male diagnosed by biopsy, with neurological signs and symptoms 60 days prior to surgery. Computerized tomography show a hyperdense expanding lesion located in cerebellum, suggesting glioma. Histopathological examination showed numerous S. mansoni ova involved by granulomatous inflammation in necrotic-exudative phase, located mainly in the internal, granular layer of the cerebellum, creating a pseudotumor in the cerebellar vermis and a recent hemorrhage in the trunk. The areas of granulomas were measured.
\end{abstract}

Key-words: Schistosoma mansoni. Schistosomiasis of cerebellum. Cerebellum tumor. Neuroschistosomiasis mansoni.

A esquistossomose do sistema nervoso central (SNC) é relativamente rara $^{18}$. Ocorre principalmente na medula espinha ${ }^{10}$, sendo responsável por cerca de $\operatorname{seis}^{27}$ a $13,4 \%{ }^{7}$ das mielopatias nas áreas endêmicas, não ultrapassando de 200 o número de casos descritos na literatura ${ }^{17}$.

A localização intracraniana, notadamente nos hemisférios cerebrais, é bem mais rara ${ }^{21}$.

0 mesmo acontece com a localização cerebelar. Até 0 momento tinham sido descritos cerca de onze casos de forma tumoral nesta localização. Este seria, portanto, o décimo segundo caso da literatura e o primeiro em que se determina a área dos granulomas.

\section{RELATO DE CASO}

Paciente de 15 anos, masculino, proveniente de Capelinha/MG. Internou-se no Hospital Santana, BH, em 05/11/03. Há 60 dias com cefaléia esporádica e fraqueza dos membros inferiores e há 15 dias com quadro de vômitos em jato e tonteira. Apresentava ataxia locomotora, sinal de Romberg com queda para traz, pescoço em posição de Maomé. Fundo de olho: estase incipiente de papila, bilateral. Afebril, anictérico, corado, hidratado, consciente e lúcido. Ausência de hepatesplenomegalia. Leucograma: 4.200 leucócitos por $\mathrm{mm}^{3}$ com eosinofilia de $11 \%$. Exame de fezes negativo.

\footnotetext{
1. Departamento de Anatomia Patológica e Medicina Legal da Faculdade de Medicina da Universidade Federal de Minas Gerais, Belo Horizonte, MG. 2. Laboratório Tafuri de Patologia, Belo Horizonte, MG. 3. Faculdade de Medicina de Ciências Médicas de Minas Gerais, Belo Horizonte, MG. 4. Hospital Santana de Belo Horizonte, MG. 5. Departamento de Patologia Geral do Instituto de Ciências Biológicas da Universidade Federal de Minas Gerais, Belo Horizonte, MG. Endereço para correspondência: Prof. Pedro Raso. Rua São Paulo 893, sala 1008, 30170-131 Belo Horizonte, MG.

Tel: $55313273-2503$

e-mail: latafuri@terra.com.br

Recebido para publicação em 23/1/2006

Aceito em 31/3/2006
} 
Em 12/11/03 foi feita a remoção microcirúrgica de massa expansiva, irregular, de limites imprecisos, ao nível do quarto ventrículo, estendendo-se da ponte ao vermis cerebelar, diagnosticada pela tomografia computadorizada como glioma.

0 paciente evoluiu mal, vindo a falecer no dia 14/11/03 com parada cardiorespiratória súbita, com impressão diagnóstica de edema de tronco encefálico.

Exames anatomopatológicos. Foram recebidos, para exames, um fragmento da ponte, pardo-amarelado e macio, medindo $1,0 \times 0,7 \times 0,7 \mathrm{~cm}$ e vários fragmentos do cerebelo, irregulares, acastanhados e macios, com área de hemorragia, medindo em conjunto $3,0 \times 2,5 \times 1,0 \mathrm{~cm}$. 0 material foi fixado em formol a $10 \%$, incluído em parafina e corado com hematoxilina e eosina (HE).

A análise morfométrica dos granulomas do cerebelo foi realizada em uma lâmina de cada amostra, coradas pela HE, segundo Caliari ${ }^{9}$, com o auxílio do analisador de imagens Koton Eletronic Versão 2.0 (software KS 300). As imagens foram captadas por câmara digital acoplada em um microscópio (aumento de 110x) que, por sua vez, estava ligado a um computador. As mensurações foram feitas considerando-se vinte campos aleatórios ou área de 640 X 480 píxels na tela do monitor, na unidade de área em micrômetros quadrados $\left(\mathrm{um}^{2}\right)$. Após o estabelecimento dos campos e captação das imagens, cada granuloma foi delimitado manualmente com o cursor e o computador determinava automaticamente 0 valor da área do granuloma.

Os cortes histológicos efetuados mostraram numerosos granulomas esquistossomóticos, na grande maioria localizados na camada interna (granular) e poucos na camada externa (molecular) do córtex cerebelar, volumosos, freqüentemente confluentes e enfileirados, centrados ou não por um ovo, em geral calcificado. Quase todos estavam na fase necróticoexsudativa caracterizados por extensa área de necrose central, envolvida por células epitelióides, semelhantes aos granulomas hepáticos descritos por Raso e Neves ${ }^{24}$, entre 45 a 70 dias de evolução da forma aguda toxêmica da esquistossomose. Diferenciavam dos granulomas do fígado e de outras sedes, nessa fase da doença, por não apresentarem eosinofilia em torno da necrose e por envolverem ovos quase sempre calcificados (Figuras 1, 2 e 3). Raros eram os granulomas no início da fase produtiva, contendo células gigantes de tipo corpo estranho. Devido à confluência e o grande volume dos granulomas, esses não só destruíram grande parte da camada granular, mas também comprimiram, desorganizaram e destruíram parte da zona intermediária (entre a camada granular e a molecular), onde se concentram as células de Purkinge.

Nas proximidades, mas independentes dos granulomas, foi comum o encontro de hiperemia, edema e focos de hemorragia na camada molecular e na zona intermediária, envolvendo vasos. Esses mostravam necrose de tipo fibrinóide da parede, provavelmente provocada por imunocomplexos. Por vezes, pequenos focos de infiltrado de células mononucleares e hemorragia recente foram identificados também em torno de vasos da leptomeninge. Não foram encontrados vermes ou resquícios deles nos locais das lesões.

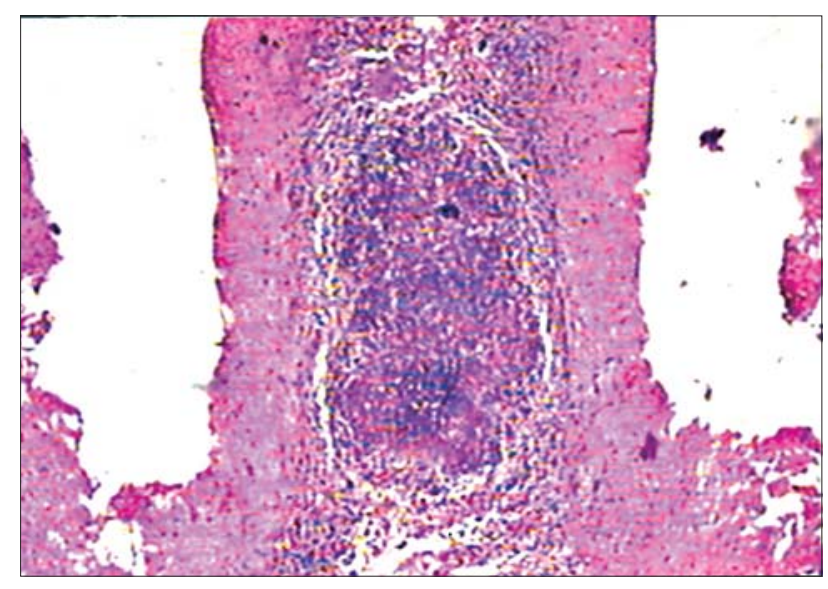

Figura 1 - Granuloma esquistossomótico na fase necrótico-exsudativa, na camada interna, granular do cerebelo. $40 x$. H\&E.

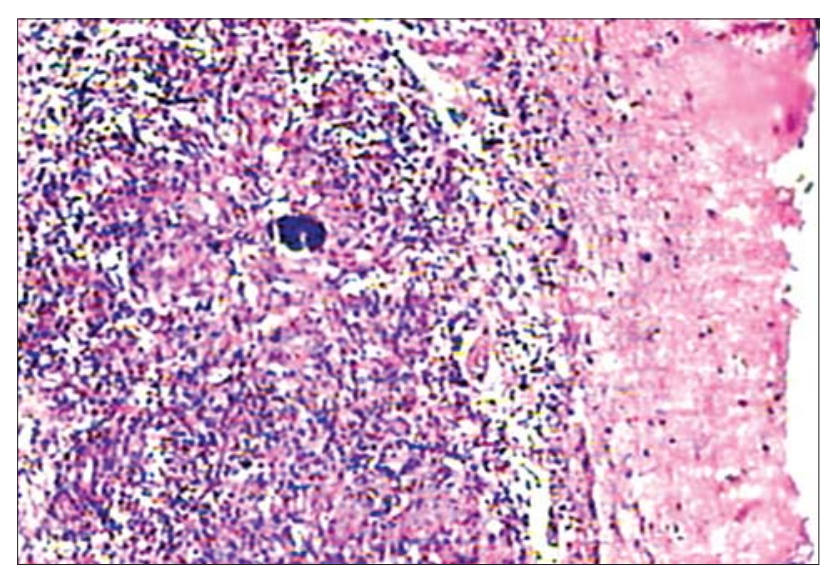

Figura 2 - Dois granulomamas confluentes, um deles centrado por ovo calcificado, na camada interna, granular do cerebelo. 220x. H\&E.

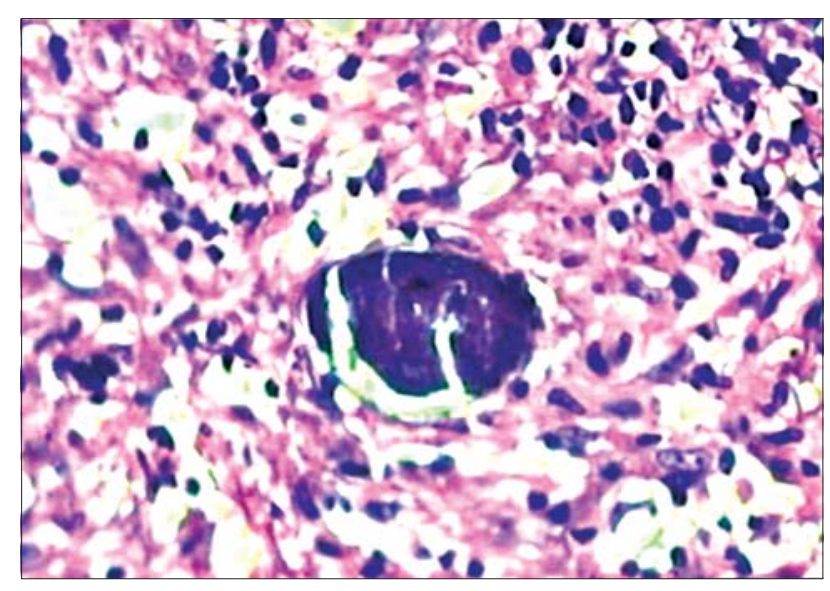

Figura 3 - Ovo de Schistosoma mansoni, calcificado, envolvido por células mononucleares e fibras colágenas. 440x. H\&E.

Os resultados da morfometria dos granulomas, sumariados na Tabela 1, mostraram que os granulomas, praticamente todos na fase necrótico-exsudativa, mediram, em média, 212.791,24 $\mathrm{mm}^{2}(+/-40.235,23)$.

Ao nível da ponte os neurônios estavam bem preservados. As lesões eram representadas por hiperemia, edema e múltiplos focos de hemorragia, por vezes extensos, preferencialmente perivasculares. Ausência de ovos e de granulomas. 
Tabela 1 - Medidas dos granulomas (aumento utilizado 110x).

\begin{tabular}{lc}
\hline Granuloma & Área $\left(\mathbf{m m}^{2}\right)$ \\
\hline 1 & $177.949,00$ \\
2 & $160.671,00$ \\
3 & $220.594,00$ \\
4 & $140.813,00$ \\
5 & $266.725,00$ \\
6 & $222.676,00$ \\
7 & $245.476,00$ \\
8 & $149.178,00$ \\
9 & $218.481,00$ \\
10 & $230.891,00$ \\
11 & $235.857,00$ \\
12 & $176.928,0$ \\
13 & $244.605,00$ \\
14 & $247.828,00$ \\
15 & $255.186,00$ \\
16 & $246.373,00$ \\
17 & $177.220,00$ \\
Média & $212.791,24$ \\
Desvio Padrão & $40.235,23$ \\
\hline &
\end{tabular}

\section{DISCUSSÃo}

O ovo, principal responsável pelas lesões anatomopatológicas na esquistossomose, independentemente da sua forma clínica, atinge a medula ou o cérebro por dois caminhos fundamentais: pela deposição in situ em consequiência da migração anômala da fêmea adulta ou pela embolização de ovos por via arteriolar, através do plexo venoso retrógrado perivertebral avalvular de Batson que comunica as veias intrabdominais com as veias da medula espinhal, favorecido pelo aumento da pressão intrabdominal durante o esforço para defecação ou acessos de tosse ${ }^{18}$. Esse mecanismo explicaria, também, a maior incidência das lesões na região lombosacra da medula.

Em quase todos os casos sintomáticos da neuroesquistossomose, o envolvimento do SNC pode se estabelecer nos primeiros estágios da infecção (forma aguda toxêmica ${ }^{16}$ ou não toxêmica ${ }^{628}$ ), no decurso da forma intestinal ou, nos portadores da forma hepatesplênica (HE), principalmente quando está associada à forma cardiopulmonar (CP) ${ }^{12}$.
Como nas formas HE e CP os ovos são carreados como êmbolos, através da via arterial ou do fluxo venoso retrógrado, as lesões se formam ao acaso, podem ser mínimas, esparsas, sem sintomatologia própria ou oligossintomática, constituindo, não raro, mero achado de necropsia ${ }^{19}$. Outras vezes, sobretudo quando é grande o número de ovos e granulomas concentrados em uma determinada área do SNC, pode surgir o efeito de massa, traduzido na tomografia computadorizada, nesse caso, por lesão nodular, captante, na região central da ponte, estendendo-se ao vermis cerebelar, sugerindo a hipótese de glioma ou meduloblastoma.

0 segundo mecanismo ${ }^{19}$, seria o da deposição de ovos pela fêmea adulta que migra para o SNC. Esse seria 0 principal, senão o único mecanismo da parasitose do SNC nos pacientes com a forma intestinal ou hepatointestinal.

Estamos de acordo com estas observações, ou seja, que o efeito de massa é produzido pela grande concentração de ovos e granulomas em uma área circunscrita da medula espinhal, do cérebro ou do cerebelo. Esse fato explicaria a formação do pseudotumor pela confluência dos granulomas e dos graves sintomas e sinais clínicos observados na maioria dos pacientes. A não demonstração dos vermes ou de seus resquícios nas lesões não invalida a hipótese de migração e postura de ovos pela fêmea, in situ. A raridade desse mecanismo explicaria também a baixa frequiência da forma tumoral da esquitossomose, principalmente no cérebro e cerebelo.

Segundo dados recentes foram levantados cerca de 18 casos de forma pseudotumoral da esquistossomose intracraniana, dos quais onze ocorreram no cerebelo. Este seria, portanto, o décimo segundo caso da literatura, com formação de massa tumoral simulando glioma e o primeiro acompanhado de hemorragia da ponte (Tabela 2).

Quase todos ocorreram em jovens do sexo masculino. Apenas dois pacientes tinham mais de 38 anos $^{313}$. Estes dois últimos não residiam em zona endêmica, mas foram contaminados no Iêmen ${ }^{13}$ e no Quênia ${ }^{3}$. Sete dos doze casos foram diagnosticados em Minas Gerais, Brasil. Apenas este caso descrito evoluiu para o óbito.

Em comum com o caso descrito por Bambirra e $\operatorname{cols}^{4}, 0$ primeiro de localização cerebelar, destaca-se a disseminação miliar maciça de ovos com formação de granulomas com extensas áreas

Tabela 2 - Forma tumoral da esquistossomose mansoni cerebelar. Revisão da literatura.

\begin{tabular}{|c|c|c|c|c|c|c|c|c|}
\hline Caso & Autores & Genêro & Idade & Ano & Localização & Granulomas & Evolução & Origem do caso \\
\hline 1 & Bambirra cols $^{4}$ & M & 20 & 1984 & hemisf. dir. & necrótico-exsudativos & Boa & Belo Horizonte \\
\hline 2 & Cabral \& Pitella & $\mathrm{F}$ & 35 & 1989 & hemisf. dir. & produtiva cura por fibrose & Boa & Belo Horizonte \\
\hline 3 & Britto cols ${ }^{6}$ & M & 21 & 1993 & hemisf. dir. & $\mathrm{NI}^{*}$ & Boa & Recife \\
\hline 4 & Lee $\operatorname{cols}^{13}$ & $\mathrm{~F}$ & 40 & 1995 & Vermis cerebelar & necrótico-exsudativa & Boa & Coréia (infectado no Iêmen) \\
\hline 5 & Pitella cols ${ }^{21}$ & M & 31 & 1996 & Vermis cerebelar & várias fases & Boa & Belo Horizonte \\
\hline 6 & Pitella cols ${ }^{21}$ & M & 11 & 1996 & hemisf. esq. & fase produtiva & Boa & Belo Horizonte \\
\hline 7 & Ferreira cols ${ }^{11}$ & M & 27 & 1998 & hemisf. dir. & diversas fases de evolução & Boa & São Luiz \\
\hline 8 & Alves cols ${ }^{2}$ & NI & 20 & 2000 & hemisf. esq.. & $\mathrm{NI}^{*}$ & Boa & Araxá \\
\hline 9 & Lesprit $\operatorname{cols}^{14}$ & NI & NI & 2001 & hemisf. esq. & necrótico-exsudastiva & Boa & França \\
\hline 10 & Braga cols 5 & M & 15 & 2003 & Lobo médio direito & produtiva & Boa & Belo Horizonte \\
\hline 11 & Amorosa cols ${ }^{3}$ & M & 38 & 2005 & cerebelo & exsudativa & Boa & América do Norte (safári no Quênia) \\
\hline 12 & Raso $\operatorname{cols}^{25}$ & M & 15 & 2006 & hemisf. hemo da Ponte & necrótico-exsudativa & Morte & Belo Horizonte \\
\hline
\end{tabular}

* NI: Não informado; hemisf.: hemisfério; dir.: direito; esq.: esquerdo; hemo: hemorragia. 
de necrose, envolvidos por macrófagos, linfócitos e poucos eosinófilos, aproximadamente no mesmo estágio evolutivo, freqüentemente contíguo, não relacionado com a forma $\mathrm{HE}$ da esquistossomose, indicando pertencer a uma mesma postura ou a posturas bem próximas umas das outras ${ }^{23}$. Este fato falaria a favor da hipótese da migração e postura dos ovos pela fêmea, in situ, apesar da não demonstração da presença de vermes nas lesões.

A tendência dos granulomas a se disporem um ao lado do outro, formando uma fileira indiana, seria o substrato anatômico da imagem linear e nodular arborizada, descrita em três casos de esquistossomose do $\mathrm{SNC}^{26}$.

Como assinalamos, em nossos resultados, não foram encontrados ovos ou granulomas ao nível da ponte. Nos fragmentos examinados havia hemorragias perivasculares, ligadas, provavelmente à necrose de arcos das paredes dos vasos, provocados por imunocomplexos. Hemorragias cerebrais, cerebelares e meníngeas foram descritas na esquitossomose do $\mathrm{SNC}^{1} 15202225$.

\section{REFERÊNCIAS BIBLIOGRÁFICAS}

1. Abath GM, Abath FGC, Silva RG. Esquistossomose encefálica associada à outras lesões ectópicas. Neurobilogia 48:3-18, 1985.

2. Alves MFC, Almeida CC, Prandini MN. Esquistossomose cerebelar: relato de caso. Jornal Brasileiro de Neurocirurgia 11:29-32, 2000.

3. Amorosa V, Kremens D, Wolfe MS, Flanigan T, Calill KM, Judy K, Kasner S, Blumberg E. Schistosoma mansoni in family 5 years after safari (Letter). Emerging Infections Diseases 11: 1-6, 2005.

4. Bambirra EA, Andrade JS, Cesarini I, Rodrigues PA, Drummond CASA. The tumoral form of shistosomiasis: report of a case with cerebellar involvement. American Society of Tropical Medicine and Hygiene 33:76-79, 1984

5. Braga BP, Costa Junior LB, Lambertucci JR. Magnetic resonance imaging of cerebellar schistosomiasis mansoni. Revista da Sociedade Brasileira de Medicina Tropical 36: 635-636, 2003.

6. Brito DMM, Filho HRCA, Furtado GJD, Filho GSC, Almeida NS, Melo RV. Esquistossomose cerebelar: relato de caso. Neurobiologia 56: 69-72, 1993.

7. Brito JCF, Silva JAG, Silva EB, Viana NO. Neuroesquistossomose medular: avaliação clínica de 5 anos. Arquivos de Neuro-Psiquiatria 50: 207-211, 1992.

8. Cabral G, Pittella JEH. Tumoral form of cerebellar schistosomiasis mansoni: report of a surgically treated case. Acta Neurochirurgica 99:148-151, 1989.

9. Caliari MV. Princípios de morfometria digital: KS 300 para iniciantes. Belo Horizonte, 1997.

10. Ferrari TC, Moreira PR, Cunha AS. Spinal cord Schistosomiasis: a prospective study of 63 cases emphasizing clinical and therapeutic aspects. Journal of Clinical Neuroscience 11: 246-253, 2004.
11. Ferreira LA, Lima FLC, Anjos MRO, Costa JML. Forma tumoral encefálica esquistossomótica: apresentação de um caso tratado cirurgicamente. Revista da Sociedade Brasileira de Medicina Tropical 31: 89-93, 1998.

12. Gonçalves EC, Fonseca AP, Pittella JEH. Frequency of schistosomiasis mansoni, of its clinicalpathological forms and the ectopic locations of the parasit in autopsies in Belo Horizonte, Brasil. Journal Tropical Medicine Hygiene 98: 289-295, 1995.

13. Lee YK, Choi TY, Jin SY, Lee DW. Imported CNS Schistosomiasis: a case report. Journal Korean Medicine Science 10: 57-61, 1995.

14. Lesprit P, Adle-Biassette H, Liance M, Nahum R, Brugieres P, Keravel Y. Photo quiz. Clinical Infections Diseases 32: 1347,1371-1372, 2001.

15. Matosinho-França LC, Meloragno Filho R, Tenuto RA. Comprometimento cerebral na esquistossomose mansônica. Revista Paulista de Medicina 67: 223-330, 1965.

16. Neves J, Marinho RP, Raso P. Spinal cord complications of acute schistosomiasis mansoni. Transactions of the Royal Society Tropical Medicine and Hygiene 67: 782-792, 1973.

17. Paz JA, Valente M, Casella EB, Marques-Dias MJ. Spinal cord Schistosomiasis in children. Analiysis of seven cases. Arquivos de NeuroPsiquiatria 60: 224-230, 2002.

18. Pittella JEH. Neuroschistosomiasis. Brain Pathology 7: 649-662, 1997.

19. Pittella JEH. The relation between involvement of the central nervous system in schistosomiasis mansoni and the clinical forms of parasitosis. A review. Journal Tropical Medicine Hygiene 94: 15-21, 1991.

20. Pittella JEH. Vascular changes in cerebral schistosomiasis mansoni: a histopathological study of fifteen cases. American Journal of Tropical Medicine and Hygiene 34: 898-902, 1985.

21. Pittella JEH, Gusmão SNS, Carvalho GTC, Silveira RL, Campos GF. Tumoral form of cerebral schistosomiasis mansoni: a report of four cases and a review of literature. Clinical Neurology and Neurosurgery 98:15-20, 1996.

22. Pompeu F, Lacerda PRS. Subaracnoid hemorragie due to Schistosoma mansoni. A rare etiologie. Neurology 221: 203-207, 1979.

23. Raso P, Bogliolo L. Esquistossomose mansoni. In: Cunha AS (ed) Patologia Editora Savier, Universidade de São Paulo, São Paulo, p. 77-130, 1970.

24. Raso P, Neves J. Contribuição ao conhecimento do quadro anatômico do fígado na forma toxêmica da esquistossomose mansoni através de punções biópsias. Anais da Faculdade de Medicina da UFMG 22:145-165, 1965.

25. Raso P, Tafuri WL, Almeida Jr N, Rodrigues JA, Santiago JM, Rocha LF. Hemorragia cerebral maciça devido ao Schistosoma mansoni. O Hospital 65:537-551, 1964.

26. Sanelli PC, Lev MH, Gonzales RG, Schaefer PW. Unique linear and nodular MR enhancement pattern in schistosomiasis of the central nervous system: report of three patients. American Journal of Radiology 177: 1471-1474, 2001.

27. Scrimgeour EM. Non tramatic paraplegia in Northem Tanzania. British Medical Journal. 283: 975-982, 1981.

28. Urban CA, Piovesan EJ, Almeida SM, Kowacs PA, Minguetti G, Werneck LC. Esquistossomose aguda com comprometimento cerebral. Arquivos de Neuro-Psiquiatria 54: 677-682, 1996. 